\title{
LAM/TSC Cell Migration to Uterus in an Experimental Model of Lymphangioleiomyomatosis. Regulation by Anti-Epidermal Growth Factor Receptor Antibody and Rapamycin
}

\author{
Elena Lesma*, Eloisa Chiaramonte, Silvia Ancona, Anna Maria Di Giulio and Alfredo Gorio
}

Laboratory of Pharmacology, Department of Health Sciences, University of Milan, Italy

\begin{abstract}
Lymphangioleiomyomatosis (LAM) is a rare lung disease affecting almost exclusively women, characterized by the invasion and abnormal proliferation of smooth muscle-like cells in pulmonary parenchyma and axial lymphatics. LAM cells bear mutations in Tuberous Sclerosis Complex (TSC) genes. It has been hypothesized that uterus might be the primary site of origin and one of the most frequent metastatic or disseminated site of LAM cells. We developed a mouse model to study the migratory and invasive properties of human LAM/TSC cells to the uterus. We also examined the action of rapamycin and anti-Epidermal Growth Factor Receptor (EGFR) antibody.
\end{abstract}

LAM/TSC cells were endonasally administered to 3 week old immunodeficient female nude mice. 5 months later mice were divided in 4 groups: control, LAM/TSC cell-administered mice, LAM/TSC cell-administered mice treated with rapamycin, and LAM/TSC cell-administered mice treated with anti-EGFR antibody. Drugs were administered for one months. Uteri were analysed for the presence of human LAM/TSC cells by COX IV antibody, lymphangiogenesis by LYVE 1 expression and angiogenesis by counting blood vessels.

LAM/TSC cells migrated to the uterus without causing any morphological lesion. Interestingly, LAM/TSC cells increased the number of blood vessels while did not cause any alteration in lymphatics vessels. Anti-EGFR antibody and rapamycin reduced the number of human LAM/TSC and counteracted the proliferation of blood vessels in uteri. Although both drugs did not change the expression of LYVE 1, localization of lymphatics was mainly in the perimetrium after drug treatment.

Our data describe the strong invasive capability of human LAM/TSC cells which migrated to the uterus. LAM/ TSC cells presence is accompanied by increased angiogenesis. Anti-EGFR antibody and rapamycin were effective in reducing the LAM/TSC cell number and blood vessel proliferation.

Keywords: Lymphangioleiomyomatosis; Tuberous sclerosis complex; Migration; Uterus; Tuberin-null cells; Mouse model

Abbreviations: LAM: Lymphangioleiomyomatosis; TSC: Tuberous Sclerosis Complex; EGF: Epidermal Growth Factor

\section{Introduction}

Lymphangioleiomyomatosis (LAM) is a rare progressive lung disease that affects almost exclusively women of childbearing age [1]. LAM can be sporadic or associated to Tuberous Sclerosis Complex (TSC), an autosomal dominant syndrome caused by mutations in TSC1 or TSC2 genes which encode hamartin and tuberin [2,3]. Hamartin and tuberin form heterodimers that inhibit the small GTPase Ras homologue enriched in brain (Rheb) [4]. The activation of Rheb leads to the activation of mammalian Target of Rapamycin (mTOR) complex 1 (TORC1) which controls protein translation, cell size, and cell proliferation $[5,6]$. The loss of tuberin or hamartin causes hyperactivation of mTORC1, which has been observed in TSC and LAM cells. LAM is characterized by proliferation of abnormal neoplastic smooth muscle cells (LAM cells) leading to lymphatic abnormalities, cystic formation in lung parenchyma, pneumothorax, and progressive pulmonary failure $[7,8]$. It is not clear whether uterine lesions, such as leiomyomas, are common manifestations in LAM and there are lacking informations regarding gynecologic pathology in LAM $[9,10]$. However, the female predominance of LAM, the reports of symptom mitigation in LAM patients after oophorectomy and worsening of symptoms during pregnancy suggests that estrogen may promote the metastasis of tuberin-null cells [11]. Moreover, uterus or adjacent areas in the retroperitoneum or pelvic cavity have been proposed as the primary site of origin of LAM [7]. LAM cells behave as cancer cells in term of growth, proliferation, cell survival in the circulation and metastasis $[12,13]$. Despite their apparently benign morphology, LAM cells exhibit metastatic properties that can be attributed to the modification caused by impaired TSC1 or TSC2 functionality [14]. Moreover, cells bearing loss of heterozigosity for TSC genes have been isolated from blood of sporadic LAM patients supporting, besides a common origin for the two diseases, the metastatic spread among organs [13].

We have isolated LAM/TSC cells from chylous thorax of a patient affected by LAM associated to TSC, bearing a germline TSC 2 mutation and an epigenetic defect causing the absence of tuberin [15]. The proliferation of these LAM/TSC cells is Epidermal Growth Factor (EGF)-dependent and blockade of EGF receptor causes cell death as we

*Corresponding author: Elena Lesma, Laboratory of Pharmacology, Department of Health Sciences, University of Milan, via di Rudinì, 820142 Milan, Italy, Tel: +39.02.503.23038; E-mail: elena.lesma@unimi.it

Received February 28, 2014; Accepted March 26, 2014; Published March 28, 2014

Citation: Lesma E, Chiaramonte E, Ancona S, Di Giulio AM, Gorio A(2014) LAM/TSC Cell Migration to Uterus in an Experimental Model of Lymphangioleiomyomatosis. Regulation by Anti-Epidermal Growth Factor Receptor Antibody and Rapamycin. J Cytol Histol S4: 007. doi:10.4172/2157-7099.S4-007

Copyright: @ 2014 Lesma E, et al. This is an open-access article distributed under the terms of the Creative Commons Attribution License, which permits unrestricted use, distribution, and reproduction in any medium, provided the original author and source are credited. 
Citation: Lesma E, Chiaramonte E, Ancona S, Di Giulio AM, Gorio A (2014) LAM/TSC Cell Migration to Uterus in an Experimental Model of Lymphangioleiomyomatosis. Regulation by Anti-Epidermal Growth Factor Receptor Antibody and Rapamycin. J Cytol Histol S4: 007. doi:10.4172/2157-7099.S4-007

previously showed in other human cells lacking-tuberin purified from angiomyolipomas, such as the TSC2 ${ }^{-1-}$ and TSC $^{-/ m e t h}$ ASM cells [16-18]. Differently, rapamycin, the specific inhibitor of mTOR, blocks the proliferation of LAM/TSC cells indicating a heterogeneous capability to affect TSC2-deficient cell proliferation. LAM/TSC cells display also the ability to survive independently from adhesion, show an extremely low proliferation rate in nonadherent condition consistent with tumour stem cell characteristics and bear characteristics of stemness thus all together these properties suggest that LAM/TSC cells might have strong invasive features. We developed a mouse model aimed at understanding such characteristics where were administered endonasally to nude mice. There we report that LAM/TSC cells migrate through the lymphatic and blood systems, reach the uterus and colonize. Moreover to test therapeutic approaches, we evaluated the effect of anti-EGFR antibody and rapamycin.

\section{Materials and Methods}

\section{Cell culture}

LAM/TSC cells were obtained from chylous effusion of a female patient affected by LAM associated to TSC and isolated, characterized and grown as previously described [15]. LAM/TSC patient who had given written informed consent according to the Declaration of Helsinki. The study was approved by the Institutional Review Board of Milan's San Paolo Hospital. The cells were maintained in culture medium contained a 50/50 mixture of DMEM/ Ham F12 (Euroclone, Paignton, UK) supplemented with $200 \mathrm{nmol} / \mathrm{L}$ hydrocortisone (SigmaAldrich, St. Louis, MO), $10 \mathrm{ng} / \mathrm{mL}$ epidermal growth factor (EGF; Sigma-Aldrich), $1.6 \mathrm{~mol} / \mathrm{L}$ ferrous sulfate (Sigma-Aldrich), and $15 \%$ fetal bovine serum (Euroclone) in a humified incubator in $5 \% \mathrm{CO}_{2}$ at $37^{\circ} \mathrm{C}$. LAM/TSC cells were used at 15 to 20 passages. As previously reported, these cells can be grown as a stabilized cell line and were checked routinely for morphological, biochemical, and genetic features.

\section{Animal experiments and pharmacological treatments}

Immunodeficient female nude mice nu/nu Hsd: athymic were obtained from Harlan Laboratories (Udine, Italy). Mice were housed in individual plastic cages under controlled conditions (temperature $22-23^{\circ} \mathrm{C}$ with light/dark cycle of $12 \mathrm{~h}$ ). All experimental procedures were performed in accordance with the Italian Guidelines for Laboratory Animals, which conforms to the European Committees Directive of November 1986 (86/609/EEC), and study protocols were previously approved by the Ministero della Sanità (2/2011 Protocol). After light anesthesia by intramuscular injection of $4 \%$ chloral hydrate, $2 \times 10^{5} \mathrm{LAM} / \mathrm{TSC}$ cells were endonasally administered to each immunodeficient female nude mouse ( 3 weeks old) using a microtip and allowing the mice to breath the drop $(25 \mu \mathrm{l})$ of physiological solution $(0.9 \% \mathrm{NaCl})$ containing the cells. At 5 months post endonasal cell administration, mice were randomly divided into four different groups: (a) LAM/TSC mice, (b) LAM/TSC mice treated with antiEGFR antibody (Merck, Darmstadt, Germany), (c) LAM/TSC mice treated with $4 \mathrm{mg} / \mathrm{kg}$ rapamycin (Rapamune-Sirolimus; Wyeth Europe, Maidenhead, UK) (d) WT control mice, treated with the vehicle. Drugs were administrated intraperitoneally (i.p) twice weekly for 1 month. Anti-EGFR antibody was administrated at a starting dose of $400 \mathrm{mg} / \mathrm{m}^{2}$ followed by a subsequent dose of 250 $\mathrm{mg} / \mathrm{m}^{2}$ and rapamycin was administrated at $4 \mathrm{mg} / \mathrm{kg}$. 3 or 6 months after endonasal administration mice were sacrificed under $4 \%$ chloral hydrate anesthesia. Uteri were excised, fixed in $4 \%$ paraformaldehyde at $4^{\circ} \mathrm{C}$ overnight and embedded in paraffin.

\section{Histology}

$8 \mu \mathrm{m}$ thick sections from paraffin-embedded samples were stained with hematoxylin and eosin for histological analysis. Images of uterus tissue sections were acquired with LEICA DM4000 B microscope (Leica microsystems $\mathrm{GmbH}$, Wetzlar, Germany) under X20 magnification. Blood vessels were hand-counted by three independent observers under light microscope (X20) in hematoxyl-eosin nonconsecutive sections. The results were expressed as the mean number of vessels per fields in each group [19,20].

\section{Immunohistochemical analysis}

Immunohistochemical analysis was performed according to the staining procedure provided by ABC-AP Vector Laboratories kit (Vector Laboratories, Burlingame, CA). Sections were deparaffinized, rehydrated through a graded ethanol series and heated to $95^{\circ} \mathrm{C}$ for 20 minutes in citrate buffer ( $\mathrm{pH}$ 6.0) for antigen retrieval. After cooling to room temperature, sections were incubated with $0.3 \% \mathrm{H}_{2} \mathrm{O}_{2} /$ methanol for 20 minutes to quench endogenous peroxidase activity and blocked in $3 \%$ bovine serum albumin/TBS solution at room temperature for 2 hours. Subsequently, the slides were incubated overnight at $4^{\circ} \mathrm{C}$ in a moist chamber with primary antibody rabbit monoclonal antibody anti-COX IV (3E11), reactive with human-specific COX $\mathrm{IV}$, the terminal enzyme complex in the respiratory chain localized to the inner mithocondrial membrane (Cell Signaling Technology, Beverly, MA; 1:350 dilution) [21] and then with the biotinylated rabbit secondary antibody (1:100; Pierce, Rockford, IL, USA). The bound secondary antibody was amplified with ABC-AP Vector Laboratories. Vector Red substrate was used for color development and nuclei were counterstained with Mayer's hematoxylin, For quantification of TSC2 human cells in uteri, the number of COX IV positive cells was calculated in 6 field for each group at X40 magnification. The results were expressed as ratio of $\mathrm{n}^{\circ}$ positive/( $\mathrm{n}^{\circ}$ total $\left.\times 10^{-3}\right)$ cells.

\section{Immunofluorescence analysis}

Sections were blocked with $3 \%$ bovine serum albumin/TBS at room temperature for 2 hours and incubated overnight at $4^{\circ} \mathrm{C}$ in a moist chamber with goat polyclonal antibody LYVE 1 (R\&D System, Minneapolis, MN, USA; $5 \mu \mathrm{g} / \mathrm{mL}$ ). Staining was revealed with goat secondary antibody Alexa Fluor 488-conjugated (Molecular Probes; Invitrogen, Paisley UK; 1:200 dilution). Nuclei were stained with $2 \mu \mathrm{g} /$ mL DAPI (Sigma-Aldrich). Samples were analyzed using a fluorescence microscope (LEICA 4000B). Lymphatic Vessels Density (LVD) was quantified using Lymphatic Vessel Analysis Protocol (LVAP) plugin for ImageJ software (National Institutes of Health, Bethesda, MD; available at http://rsb.info.nih.gov/ij/index. Html).

\section{Statistical analysis}

Statistical analyses were carried out by Student's t-test or one-way ANOVA analysis as appropriate to determine differences between experimental groups. All data were expressed as mean \pm SEM. In all cases, $\mathrm{P}$ values less than 0.05 were considered statistically significant. Analysis was performed with the GraphPad Prism 5 (version 5, GraphPad software, Inc., San Diego, CA).

\section{Results}

\section{Invasion of uterus by LAM/TSC cells}

The capability of LAM/TSC cells to invade uterus was studied in vivo by administrating the cells to 3 week old female nude mice when the mice have not yet reached the sexual maturation to allow 
Citation: Lesma E, Chiaramonte E, Ancona S, Di Giulio AM, Gorio A (2014) LAM/TSC Cell Migration to Uterus in an Experimental Model of Lymphangioleiomyomatosis. Regulation by Anti-Epidermal Growth Factor Receptor Antibody and Rapamycin. J Cytol Histol S4: 007. doi:10.4172/2157-7099.S4-007

them to better invade organs. No macroscopic uterine alterations were observed at 3, or 6 months after LAM/TSC cell administration (data not shown). Indeed, 3 and 6 months after cell inhalation, human LAM/ TSC cells were detected in the myometrium of mice uteri (Figure 1A). Here we showed that the human cells were detected 3 and 6 months after cell administration, by immunoreactivity to COX IV antibody. No aggregation in cluster formations was observed. Number of human cells in uteri at 3 and 6 months from LAM/TSC cell administration was $2.6 \pm 1.2$ and $3.9 \pm 0.66$ cells $/\left(n^{\circ}\right.$ total cells $\left.\times 10^{-3}\right)$, respectively (Figure $1 \mathrm{~B}$ ).

\section{Anti-EGFR antibody and rapamycin action on LAM/TSC cells in uterus}

We recently reported that LAM/TSC proliferation is blocked by rapamycin and anti-EGFR antibody administration that also causes cell death [15]. The effect of the two drugs on these cells migrated to the uterus. The quantitative and qualitative evaluations of the action of the two agents show that both significantly reduced their number (Figure 2).

Effect of anti-EGFR $\mathrm{Ab}$ and rapamycin on angiogenesis and lymphangiogenesis caused by LAM/TSC cell administration in uteri

Six months after LAM/TSC cell administration, numerous blood vessels were observed in mice uteri indicating a specific ability of LAM/ TSC cells to cause angiogenesis (Figure 2A). Both, anti-EGFR Ab and rapamycin reduced the number of blood vessels as demonstrated quantitatively by assessing the number of blood vessels per fields (Figure $3 \mathrm{~A}$ and $\mathrm{B}$ ).

Lymphatic involvement in human LAM has been demonstrated by the observation of higher number of lymphatics in the lungs and LAM lesions, and in mice lungs after inoculation of TSC2 ${ }^{-/}$ASM cells [22,23]. However, after LAM/TSC administration immunoreactivity to LYVE 1, a well known lymphatic capillary marker and lymph-specific

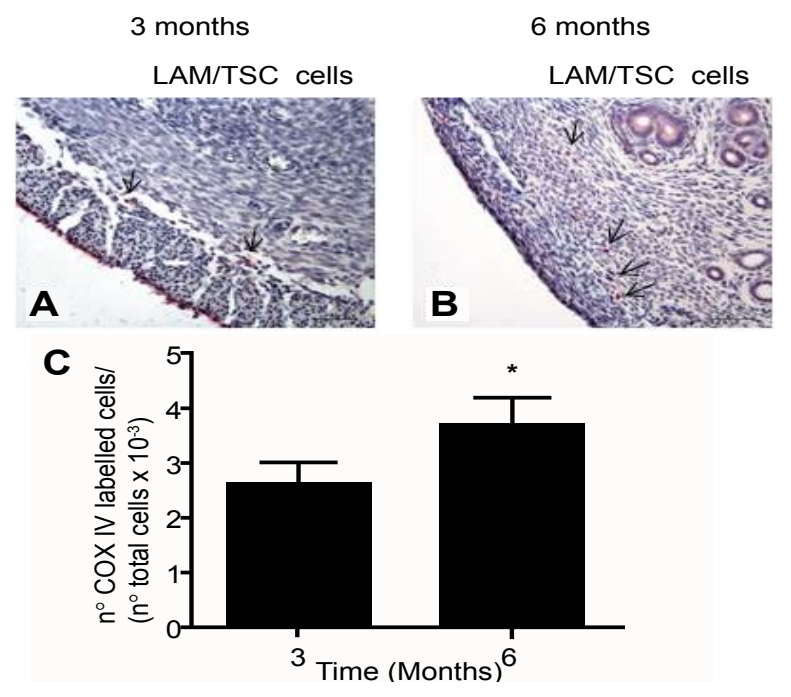

Figure 1: Immunohistochemical detection of human LAM/TSC cells by using COX IV rabbit monoclonal antibody (red staining) in uteri of mice $3(A)$ and 6 (B) months after human LAM/TSC cell administration. COX IV positive cells (arrows) are detected in the myometrium layer (X40). Scale bars: $50 \mu \mathrm{m}$. Quantification of LAM/TSC cells in uteri was calculated as percentage of COX IV-labelled LAM/TSC cells/ total number of cells in the field (C). Data are expressed as mean \pm SEM. ${ }^{*} P<0.05$ vs $n^{\circ} \mathrm{COX}$ IV labelled cells/ $\left(n^{\circ}\right.$ total cells $\times 10^{-3}$ ) of mice 3 months after LAM/TSC cell administration
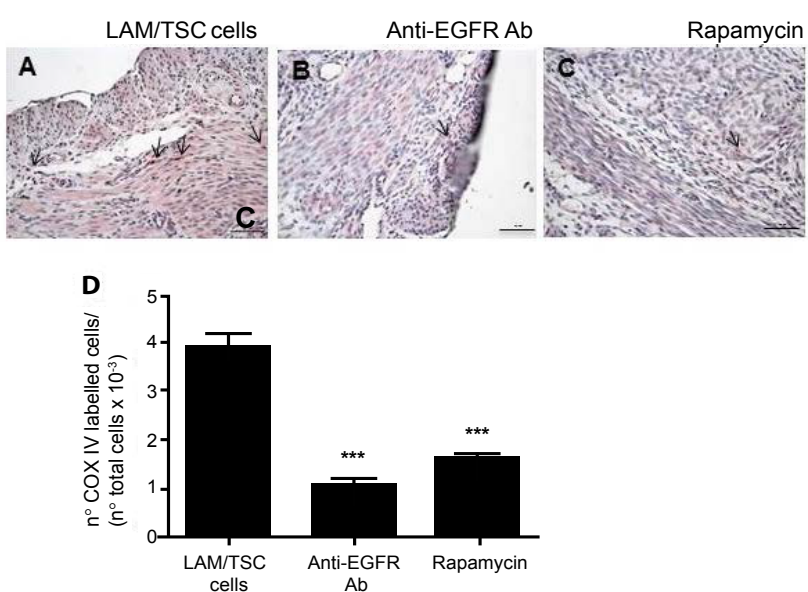

Figure 2: Immunohistochemical detection of human LAM/TSC cells in uter and anti-EGFR anitbody and rapamycin effect : detection of LAM/TSC cells by using COX IV rabbit monoclonal antibody (red staining) in uteri of mice 6 months after LAM/TSC cell administration (A), in LAM/TSC cell-administered mice treated with anti-EGFR antibody (B), and in LAM/TSC cell-administered mice treated with rapamycin (C) (X40). Scale bars: $50 \mu \mathrm{m}$. Quantification of LAM/TSC cells in uteri was calculated as percentage of COX IV-labelled LAM/ TSC cells on the total number of cells in the field (D). ${ }^{* *} P<0.001, v s n^{\circ}$ of COX IV labelled cells in LAM/TSC cell-administrated mice.

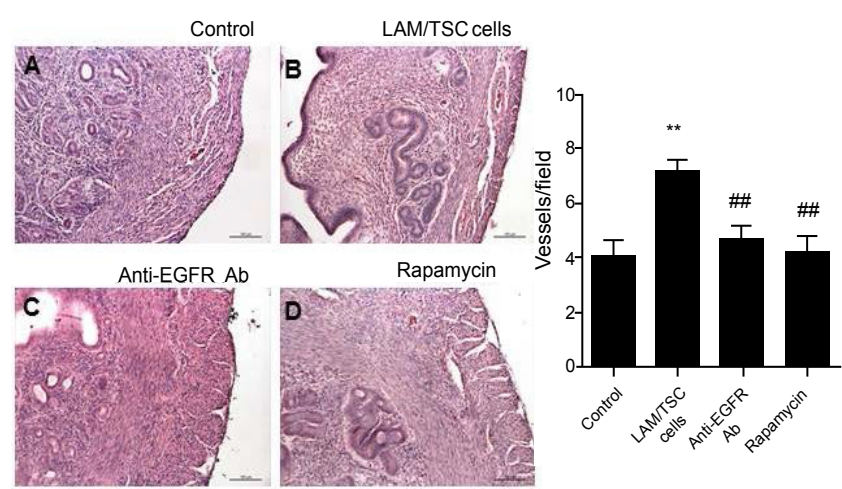

Figure 3: Analysis of angiogenesis following staining with hematoxylin and eosin in uteri of control nude mice (A), 6 months after LAM/TSC cell administration (B), following treatment with anti-EGFR $A b(C)$ or rapamycin (D). Representative images are shown (H\&E X20). Scale bars: $100 \mu \mathrm{m}$. Quantification was done by counting the number of blood vessels in a field ( 6 fields for 8 mice for each group) as described in Material and Methods (E). Data are expressed as mean \pm SEM. ${ }^{* *} P<0.01$ vs control mice. \#\#P<0.01 vs LAM/TSC-cell administered mice.

receptor for hyaluronan, was unchanged in uterus (Figure 4A) [24]. Lymphatic Vessel Density (LVD) based on LYVE 1 staining was not increased by LAM/TSC cell administration and the treatments with anti-EGFR antibody and rapamycin did not change it (Figure $4 \mathrm{~A}$ and B). In control and LAM/TSC cell-administered mice, lymphatic vessels were observed in the connective tissue or stroma between the longitudinal and circular muscle layers of the myometrium while after treatment with rapamycin or anti-EGFR antibody LYVE 1 positivity was almost exclusively localized in the perimetrium (Figure 4A).

\section{Discussion}

LAM is associated with the metastasis of histopathologically benign TSC1 or TSC2-deficient cells and has a very strong gender predisposition. LAM is a neoplasm arising from constitutive activation 


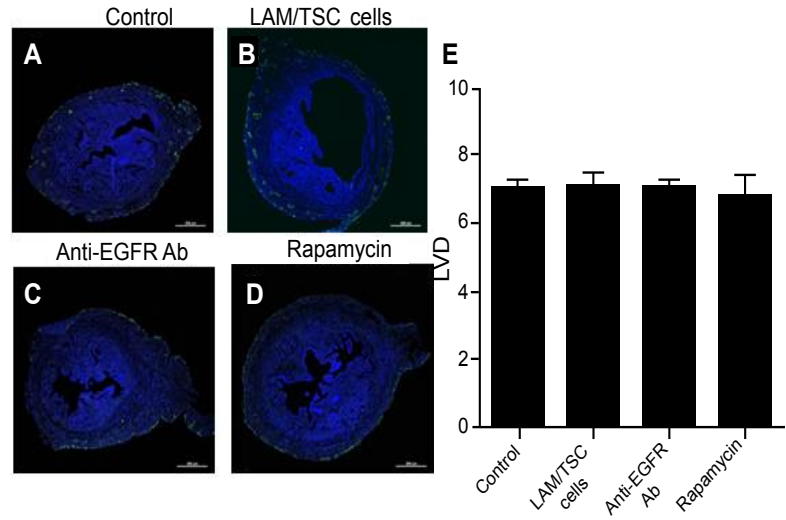

Figure 4: Analysis of lymphatic vessels in uterus sections after LAM/TSC cell administration. Representative images of uterus sections showing labelling of lymphatic capillary marker LYVE-1 (green) in control mice (A), in LAM/TSC cell-administered mice (B), in LAM/TSC cell-administered mice treated with anti-EGFR antibody (C) and in LAM/TSC cell-administered mice treated with rapamycin (D) (X5). Nuclei of cells are stained with DAPI (blue). Scale bars: $400 \mu \mathrm{m}$. Quantification of lymphatic vessels in uteri was calculated as LVD in five fields for each mouse $(E)$. Data are expressed as mean \pm SEM.

of the mTOR signaling pathway dysregulated by a functional loss of TSC genes [2,3]. Our group has contributed to elucidate some of the properties of human TSC and LAM cells including the role of epigenetic modification in causing the loss of heterozigosity, their metastatic properties described in a LAM model and the specific pharmacological actions of agents such rapamycin, anti-EGFR antibody and rosuvastatin $[15-18,25,26]$.

Recently, the generation of a variety of mouse models by the injection of TSC2-deficient cells in specific conditions has given new input to study the invasive features of TSC2-deficient cells providing useful systems for in vivo drug testing [23,27,28]. We previously reported the development of an animal model of LAM through the endonasal administration of human TSC2 $\%$ ASM cells that infiltrate lymph nodes and lungs with consequent enlargement of the alveolar spaces [23]. Rapamycin and, more specifically, anti-EGFR antibody counteract these pulmonary lesions caused by the administration of $\mathrm{TSC}^{-1-}$ ASM cells. Here we report that also the uterus may represent a target for LAM cells.

LAM/TSC cells were isolated from chylous thorax of a patient affected by LAM associated to TSC, in vitro display the ability to survive in adherent and nonadherent conditions and bear EMT features [5]. In our opinion these characteristics may help to give new insights to study the migratory ability of LAM cells in vivo and to evaluate pharmacological treatments.

LAM is a pulmonary disease characterized by abnormal proliferation of smooth muscle-like cells in the lungs and along the axial lymphatic system. Extra pulmonary LAM has major locations in the mediastinum, in the upper retroperitoneal areas close to the abdominal aorta, and the pelvic cavity [29]. However it is still unclear how often the uterus is also affected in patients with LAM. Hayashi et al. reported that women with pulmonary sporadic LAM and TSC-LAM are commonly affected by lesions in genital organs, most frequently within the uterus including diffuse leiomyomatosis, benign metastasizing leiomyoma, epithelial leiomyosarcoma, and endometrial stromal sarcoma [9]. On the other hand, another study demonstrated that uterine leiomyomas are not more common in LAM than in the general population [10]. However, the use of hysterectomy and oophorectomy in LAM has a high frequency. The mechanism for the development of uterine LAM remains unclear. An important involvement of uterus in LAM is supported by the hypothesis that the uterus may be one of the most frequent metastatic or disseminated sites of LAM and the chance that uterus might be the primary site of origin of LAM cells. Indeed, in a less aggressive rare lung disease, called benign metastasizing leiomyoma, it is well known that uterine leiomyomas metastasize to the lungs [30]. Human LAM/ TSC cells survived and migrated to uteri, and were detected 3 and 6 months after cell administration confirming in vivo the strong invasive properties of LAM cells. LAM/TSC cells were administered to 3 week old female nude mice to allow to the cells to invade during mice sexual maturation and to better resemble the LAM features such as the frequent onset at the beginning of the childbearing years. Human cells did not form clusters even if their number was higher at 6 months after cell administration. The metastatic potential of LAM cells is demonstrated by the presence of LAM cells in blood and lymphatic vessels, the evidence of LAM recurrence in transplanted lungs of recipients and the finding of identical TSC 2 mutations in lung LAM cells and renal angiomyolipoma cells of patients with sporadic LAM $[3,13,31]$. Our data support the concept that LAM is associated with metastasis of histologically benign TSC2-deficient cells. In our animal model we did not observed any macroscopic and morphological lesions in uteri. This might be ascribed to the small number of LAM/TSC cells that reached the uterus, and otherwise to the healthy condition of the animal. Changing the route of administration, such as intraperitoneal or subcutaneous administration, might give a more effective invasion. It is also possible that in a healthy mouse a hormone administration might be needed to enhance uterine lesions as it occurs in LAM progression where estrogen have a specific role [11]. LAM progression is enhanced in women during the reproductive years and estradiol promotes LAM cell proliferation and likely metastasis in vivo indicating an important role of estrogens in LAM [11]. Lung LAM lesions share many features with uterine leiomyomas such as similar appearing smooth muscle cells, expression of estrogen and progesterone receptors and proliferative sensitivity to estrogens. The fact that uterine leiomyomas may be related to a genetic TSC defect is demonstrated by the development of spontaneous uterine leiomyomas in Eker rat model with high frequency [32]. Eker rats carry a germline mutation in the rat homologue of the TSC2 gene demonstrating that the genetic defect in TSC confers susceptibility to uterine leiomyoma [32]. Likewise, a uterine-specific Tsc-2 knockout mouse develops myometrial proliferation and uterine leiomyomas with progressive uterine enlargement both prevented by ovariecomy [30]. Myometrial Tsc2 null tumors were found in the lungs suggesting the ability of Tsc2 null myometrial cells to migrate from the uterus to the lungs.

Rapamycin and anti-EGFR antibody treatment significantly reduced the number of LAM/TSC cells in uteri. Rapamycin has been shown to stabilize the lung function of LAM patients and to promote the regression of TSC angiomyolipomas which tended to increase again once the therapy was discontinued [33-35]. Anti-EGFR antibody is efficacious in treating several types of cancer such as colorectal and head-and-neck cancers [36]. We previously showed that anti-EGFR antibody more efficiently than rapamycin reversed the pulmonary alterations caused by TSC $2 \%$ ASM cell administration [23]. In this case the two agents had similar effects in reducing the number and, likely, the infiltration of LAM/TSC cells in uterus.

Uterine LAM lesions in sporadic LAM patients have abundance of lymphatic endothelial cells compared to those of TSC-LAM patients 
Citation: Lesma E, Chiaramonte E, Ancona S, Di Giulio AM, Gorio A (2014) LAM/TSC Cell Migration to Uterus in an Experimental Model of Lymphangioleiomyomatosis. Regulation by Anti-Epidermal Growth Factor Receptor Antibody and Rapamycin. J Cytol Histol S4: 007. doi:10.4172/2157-7099.S4-007

Page 5 of 6

suggesting an important and specific role for lymphangiogenesis in the progression of uterine lesions in sporadic LAM [9]. In our model, angiogenesis was increased in uteri after LAM/TSC administration while lymphangiogenesis remained unchanged. The increased number of blood vessels is related to LAM/TSC cell administration suggesting that in uteri of our mouse model the haematological circulatory system might be preferred to lymphatic system for migration and LAM /TSC cell infiltration may cause the promotion of blood vessel formation. The treatment with rapamycin and anti-EGFR antibody caused a reduction of the number of blood vessels that became similar to the control.

LAM cell clusters enveloped by lymphatic endothelial cells have been identified in LAM lesions that express lymphatic endothelial markers such as VEGFR-3, podoplanin, and prox-1 and VEGF-D is elevated in serum of LAM patients correlating with the severity of the disease [37]. However, in our model LYVE 1 positivity in uteri did not differ in quantitative expression between the groups while it changed in localization. In control and LAM/TSC cell-administeredmice LYVE 1 lymphatic vessel profile was within the myometrial circular and longitudinal muscle layers while after the administration of rapamycin and anti-EGFR antibody, immunoreactivity was mainly localized in the perimetrium. Thus both treatments affected lymphatic vessel distribution in uteri while they were effective in the control of lymphangiogenesis in lungs in our previous mouse model developed with the administration of TSC2 ${ }^{-1-}$ ASM cells [23].

These data regarding angiogenesis and lymphangiogenesis differ from what we previously observed in lungs by administration of $\mathrm{TSC}^{-1-}$ ASM cells [24]. In that case lung lesions were accompanied by an increase of lymphangiogenesis markers such as the expression of LYVE 1 and the levels of VEGF in serum and lung tissues [24]. On the contrary, angiogenesis was not altered in any experimental group. These differences might be due to the tissue characteristics, lung and uterus, and to the route of administration that favours the invasion of lungs.

In conclusion our data describe the ability of LAM/TSC cells to migrate and invade uteri such as benign cancer cells without causing any morphological alteration. In this model the administration of human cells increased the number of blood vessels while surprisingly lymphangiogenesis seems unaffected. Anti-EGFR antibody and rapamycin treatments confirm their therapeutic potential by reducing the number of human LAM/TSC cells and normalizing the number of blood vessels. Thus these data support the therapeutic value of antiEGFR antibody and rapamycin in controlling ASM cell invasion in TSC and LAM patients.

\section{Acknowledgments}

This work was supported by grants from the AiLAM Onlus (Italian LAM Patients Association), AST (Italian TSC Patient Association) and Accordo Quadro di Collaborazione tra Università Lombarde (from Lombardy Region).

\section{References}

1. Johnson SR, Tattersfield AE (2002) Lymphangioleiomyomatosis. Semin Respir Crit Care Med 23: 85-92.

2. Carsillo T, Astrinidis A, Henske EP (2000) Mutations in the tuberous sclerosis complex gene TSC2 are a cause of sporadic pulmonary lymphangioleiomyomatosis. Proc Natl Acad Sci U S A 97: 6085-6090.

3. Astrinidis A, Khare L, Carsillo T, Smolarek T, Au KS, et al. (2000) Mutational analysis of the tuberous sclerosis gene TSC2 in patients with pulmonary lymphangioleiomyomatosis. J Med Genet 37: 55-57.

4. Tee AR, Fingar DC, Manning BD, Kwiatkowski DJ, Cantley LC, et al. (2002) Tuberous sclerosis complex-1 and -2 gene products function together to inhibit mammalian target of rapamycin (mTOR)-mediated downstream signaling. Proc Natl Acad Sci U S A 99: 13571-13576.

5. El-Hashemite N, Zhang H, Henske EP, Kwiatkowski DJ (2003) Mutation in TSC2 and activation of mammalian target of rapamycin signalling pathway in renal angiomyolipoma. Lancet 361: 1348-1349.

6. Strizheva GD, Carsillo T, Kruger WD, Sullivan EJ, Ryu JH, et al. (2001) The spectrum of mutations in TSC1 and TSC2 in women with tuberous sclerosis and lymphangiomyomatosis. Am J Respir Crit Care Med 163: 253-258.

7. Taveira-DaSilva AM1, Pacheco-Rodriguez G, Moss J (2010) The natural history of lymphangioleiomyomatosis: markers of severity, rate of progression and prognosis. Lymphat Res Biol 8: 9-19.

8. Darling TN, Pacheco-Rodriguez G, Gorio A, Lesma E, Walker C, et al. (2010) Lymphangioleiomyomatosis and TSC2-/- cells. Lymphat Res Biol 8: 59-69.

9. Hayashi T, Kumasaka T, Mitani K, Terao Y, Watanabe M, et al. (2011) Prevalence of uterine and adnexal involvement in pulmonary lymphangioleiomyomatosis: a clinicopathologic study of 10 patients. Am J Surg Pathol 35: 1776-1785.

10. Taveira-Dasilva AM, Rabel A, Gochuico BR, Avila NA, Moss J (2011) Prevalence of uterine leiomyomas in lymphangioleiomyomatosis. Fertil Steril 96: 711-714.

11. Yu JJ1, Robb VA, Morrison TA, Ariazi EA, Karbowniczek M, et al. (2009) Estrogen promotes the survival and pulmonary metastasis of tuberin-null cells. Proc Natl Acad Sci U S A 106: 2635-2640.

12. Henske EP, McCormack FX (2012) Lymphangioleiomyomatosis - a wolf in sheep's clothing. J Clin Invest 122: 3807-3816.

13. Crooks DM, Pacheco-Rodriguez G, DeCastro RM, McCoy JP Jr, Wang JA, et al. (2004) Molecular and genetic analysis of disseminated neoplastic cells in lymphangioleiomyomatosis. Proc Natl Acad Sci U S A 101: 17462-17467.

14. Huang J, Manning BD (2008) The TSC1-TSC2 complex: a molecular switchboard controlling cell growth. Biochem J 412: 179-190.

15. Lesma E, Ancona S, Sirchia SM, Orpianesi E, Grande V, et al. (2014) TSC2 epigenetic defect in primary LAM cells. Evidence of an anchorage-independent survival. J Cell Mol Med

16. Lesma E, Grande V, Carelli S, Brancaccio D, Canevini MP, et al. (2005) Isolation and growth of smooth muscle-like cells derived from tuberous sclerosis complex-2 human renal angiomyolipoma: epidermal growth factor is the required growth factor. Am J Pathol 167: 1093-1103.

17. Lesma E, Grande V, Ancona S, Carelli S, Di Giulio AM, et al. (2008) Anti-EGFR antibody efficiently and specifically inhibits human TSC2-/- smooth muscle cel proliferation. Possible treatment options for TSC and LAM. PLoS One 3: e3558.

18. Lesma E, Sirchia SM, Ancona S, Carelli S, Bosari S, et al. (2009) The methylation of the TSC2 promoter underlies the abnormal growth of TSC2 angiomyolipoma-derived smooth muscle cells. Am J Pathol 174: 2150-2159.

19. Mensah-Brown EP, Rizk DE, Patel M, Chandranath SI, Adem A (2004) Effects of ovariectomy and hormone replacement on submucosal collagen and blood vessels of the anal canal of rats. Colorectal Dis 6: 481-487.

20. Rizk DE, Mensah-Brown EP, Chandranath SI, Ahmed I, Shafiullah M, et al. (2003) Effects of ovariectomy and hormone replacement on collagen and blood vessels of the urethral submucosa of rats. Urol Res 31: 147-151.

21. Li S, Thangapazham RL, Wang JA, Rajesh S, Kao TC, et al. (2011) Human TSC2-null fibroblast-like cells induce hair follicle neogenesis and hamartoma morphogenesis. Nat Commun 2: 235

22. Kumasaka T, Seyama K, Mitani K, Sato T, Souma S, et al. (2004) Lymphangiogenesis in lymphangioleiomyomatosis: its implication in the progression of lymphangioleiomyomatosis. Am J Surg Pathol 28: 1007-1016.

23. Lesma E, Eloisa C, Isaia E, Grande V, Ancona S, et al. (2012) Development of a lymphangioleiomyomatosis model by endonasal administration of human TSC2-/- smooth muscle cells in mice. Am J Pathol 181: 947-960.

24. Banerji S, Ni J, Wang SX, Clasper S, Su J, et al. (1999) LYVE-1, a new homologue of the CD44 glycoprotein, is a lymph-specific receptor for hyaluronan. J Cell Biol 144: 789-801.

25. Carelli S, Lesma E, Paratore S, Grande V, Zadra G, et al. (2007) Survivin expression in tuberous sclerosis complex cells. Mol Med 13: 166-177.

26. Lesma E, Ancona S, Orpianesi E, Grande V, Di Giulio AM, et al. (2013) Chromatin remodeling by rosuvastatin normalizes TSC2-/meth cell phenotype through the expression of tuberin. J Pharmacol Exp Ther 345: 180-188. 
Citation: Lesma E, Chiaramonte E, Ancona S, Di Giulio AM, Gorio A (2014) LAM/TSC Cell Migration to Uterus in an Experimental Model of Lymphangioleiomyomatosis. Regulation by Anti-Epidermal Growth Factor Receptor Antibody and Rapamycin. J Cytol Histol S4: 007. doi:10.4172/2157-7099.S4-007

Page 6 of 6

27. Goncharova EA, Goncharov DA, Fehrenbach M, Khavin I, Ducka B, et al. (2012) Prevention of alveolar destruction and airspace enlargement in a mouse mode of pulmonary lymphangioleiomyomatosis (LAM). Sci Transl Med 4: 154 ra134.

28. Liu F, Lunsford EP, Tong J, Ashitate Y, Gibbs SL, et al. (2012) Real-time monitoring of tumorigenesis, dissemination, \& drug response in a preclinical model of lymphangioleiomyomatosis/tuberous sclerosis complex. PLoS One 7: e38589.

29. Matsui K, Tatsuguchi A, Valencia J, Yu Zx, Bechtle J, et al. (2000) Extrapulmonary lymphangioleiomyomatosis (LAM): clinicopathologic features in 22 cases. Hum Pathol 31: 1242-1248.

30. Tietze L, Günther K, Hörbe A, Pawlik C, Klosterhalfen B, et al. (2000) Benign metastasizing leiomyoma: a cytogenetically balanced but clonal disease. Hum Pathol 31: 126-128.

31. Karbowniczek M, Astrinidis A, Balsara BR, Testa JR, Lium JH, et al. (2003) Recurrent lymphangiomyomatosis after transplantation: genetic analyses reveal a metastatic mechanism. Am J Respir Crit Care Med 167: 976-982.
32. Cook JD, Walker CL (2004) The Eker rat: establishing a genetic paradigm linking renal cell carcinoma and uterine leiomyoma. Curr Mol Med 4:813-824.

33. Bissler JJ, McCormack FX, Young LR, Elwing JM, Chuck G, et al (2008) Sirolimus for angiomyolipoma in tuberous sclerosis complex or lymphangioleiomyomatosis. N Engl J Med 358: 140-151.

34. Davies DM, de Vries PJ, Johnson SR, McCartney DL, Cox JA, et al. (2011) Sirolimus therapy for angiomyolipoma in tuberous sclerosis and sporadic lymphangioleiomyomatosis: a phase 2 trial. Clin Cancer Res 17: 4071-4081.

35. McCormack FX, Inoue Y, Moss J, Singer LG, Strange C, et al. (2011) Efficacy and safety of sirolimus in lymphangioleiomyomatosis. N Engl J Med 364: 15951606.

36. Dienstmann R, Markman B, Tabernero J (2012) Application of monoclona antibodies as cancer therapy in solid tumors. Curr Clin Pharmacol 7: 137-145.

37. Seyama K, Kumasaka T, Kurihara M, Mitani K, Sato T (2010) Lymphangioleiomyomatosis: a disease involving the lymphatic system. Lymphat Res Biol 8: 21-31.
This article was originally published in a special issue, Histology and Histopathology handled by Editor(s). Borislav A. Alexiev, University of Maryland Medical Center, USA 